\section{Das Dilemma mit dem Wirksamkeitsnachweis}

\author{
Eine abschließende Bewertung der sublingualen Immuntherapie \\ (SLIT) bei Kindern wird in der aktuellen deutschen S2-Leitlinie zur \\ Hyposensibilisierung aufgrund der unzureichenden Datenlage nicht \\ vorgenommen. Eine neuere Metaanalyse spricht nun für einen \\ eindeutigen Effekt dieser Therapieform - oder doch nicht?
}

W ie wirksam ist die SLIT bei Kindern? Obwohl in der Praxis schon breit eingesetzt, bleibt bei vielen Pädiatern angesichts der verwirrenden Studienlage zur Wirksamkeit bzw. Unwirksamkeit der SLIT bei Kindern ein ,ungutes Gefühl“. Erfreut aufgenommen wurde deshalb das Ergebnis einer aktuellen Metaanalyse von Penagos et al. (Ann Allergy Asthma Immunol 2006; 97: 141-8), in der die SLIT bei Kindern als wirksam eingestuft wurde. „Doch auch Metaanalysen können mit Fehlern behaftet sein und das ist bei der PenagosArbeit der Fall“, warnte Priv.-Doz. Dr. Thomas Hirsch, Bergen auf Rügen.

Hirsch belegte dies anhand zweier Einzelstudien, die in die Analyse eingeflossen waren. In einem Fall seien die Ergebnisse einer Untersuchung durch die Autoren der Metaanalyse komplett anders bewertet worden als durch die Autoren der Originalarbeit, in dem anderen Fall wurden primäre Wirksamkeitsendpunkte in nicht zulässiger Weise zusammen gefasst. Für beide Studien ergab sich in der Metaanalyse insgesamt ein positiveres Bild für die Wirksamkeit der SLIT bei Kindern, als es die wissenschaftliche Interpretation der Studien eigentlich erlaube, sagte Hirsch.

\section{Plazebo-SLIT vom Apotheker?}

Unter den insgesamt 18 Studien zur SLIT bei Kindern gibt es mittlerweile sieben neuere Studien mit einer größeren Zahl von Patienten und zu allen wichtigen Allergenen. Dennoch sei weiterhin kein eindeutiger Trend zu erkennen, bedauerte Hirsch. Dies liegt in erster Linie an der immer noch sehr heterogenen Methodik bzw. Qualität, mit der die Studien durchgeführt werden. „Tendenziell sehen wir lediglich, dass kleinere
Studien mit einer schlechteren Methodik zu besseren Ergebnissen führen als größere Studien mit einer adäquaten Methodik", fasste Hirsch zusammen. Seine provokante Forderung: „Bevor Sie eine SLIT für Kinder verordnen, lassen Sie lieber den Apotheker ein Plazebopräparat mixen, das hilft genauso gut!“

Das wollten viele Kollegen im Auditorium so nicht akzeptieren: Zum einen gäbe es keinen wissenschaftlichen Grund, warum die SLIT bei Kindern nicht genauso gut wirken sollte wie bei Erwachsenen. Außerdem sähen viele Pädiater in der Praxis tatsächlich eine relevante Wirkung und häufig ginge auch ein orales Allergiesyndrom unter SLIT zurück - so einige Diskussionsbeiträge. Möglicherweise wurden in den Studien vorhandene Compliance-Probleme der Kinder nicht genügend berücksichtigt oder die Sym-

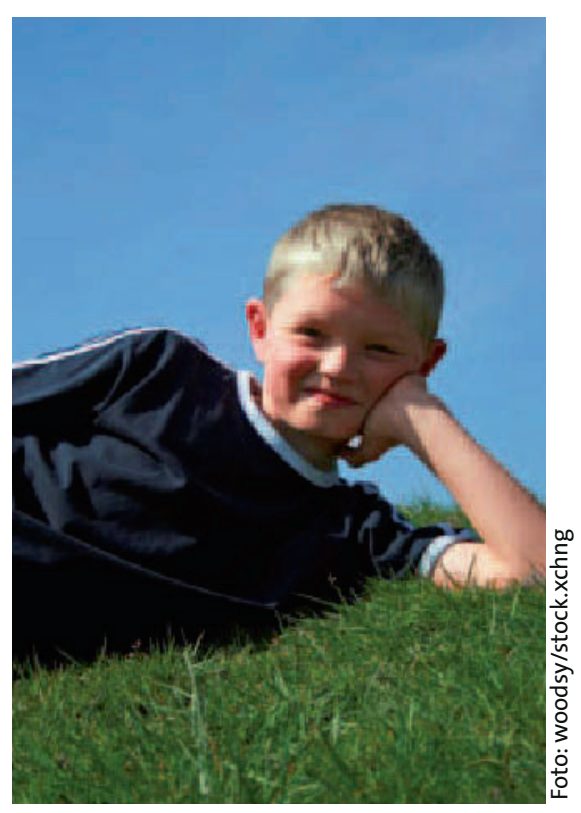

Ein Kandidat für eine sublinguale Immuntherapie? ptome der Studienteilnehmer waren so mild, dass sich in den plazebokontrollierten Studien einfach kein statistisch signifikanter Effekt nachweisen ließ.

\section{Qualitätssprung in Sicht?}

Vielleicht führt die Anfang 2007 in Kraft getretene EU-Kinderarzneimittelverordnung mit ihren neuen Regelungen zur klinischen Prüfung von Arzneimitteln als Voraussetzung für eine Zulassung bei Kindern mittelfristig zu mehr Klarheit. In dieser Verordnung werden sehr hohe Anforderungen an die Durchführung von Studien mit Kindern gestellt, erklärte Dr. Susanne Kaul vom Paul-EhrlichInstitut, Langen. In einem vorab einzureichenden Studienprüfplan muss u. a. eine detaillierte Übersicht über die geplante Vorgehensweise sowie eine genaue Auflistung des gewählten Studiendesigns, der Patientenzahl, der Ein- und Ausschlusskriterien und der Auswertungsstatistik einschließlich einer Begründung der Vorgehensweise bei den zuständigen Zulassungsbehörden eingereicht werden. Ein Expertenkomitee bewertet dann die geplante Studie. Insgesamt habe die Sicherheit bei diesem Vorgehen oberste Priorität, erläuterte Kaul. Bei der SLIT bedeutet dies z. B. die Ausarbeitung von altersstratifizierten Risikomanagement-Strategien. „Ein 15jähriger kommt mit einer Zungenschwellung unter der SLIT vielleicht noch gut zurecht, ein vierjähriges Kind wird aber sehr wahrscheinlich in Panik verfallen", erläuterte Kaul.

Nun bleibt abzuwarten, ob sich die Herstellerfirmen diesen hohen Anforderungen stellen. Viele kleinere Firmen werden die hohen Kosten für solche Studien eventuell gar nicht aufbringen können. Und größere Firmen werden sorgfältig kalkulieren, ob sich die anfallenden Studienkosten später auf ein SLIT-Präparat für Kinder umlegen lassen. Auf die Frage von Priv.-Doz. Dr. Jörg KleineTebbe, Berlin, ob bei der Kinder-SLIT denn nun das Glas halb voll oder halb leer sei, konnte keine abschließende Antwort gefunden werden.

\section{$b k$}

Symposium 12 ,Spezifische Immuntherapie im Kindes- und Jugendalter". Zweiter Gemeinsamer Deutscher Allergiekongress, Lübeck, 26.-29. September 2007 